Mots. Les langages du politique

Suisse, laboratoire politique européen?

\title{
Marques linguistiques et manipulation. Le cas d'une campagne de l'extrême droite suisse
}

Marcel Burger, Gilles Lugrin, Raphaël Micheli et Stéphanie Pahud

\section{OpenEdition}

\section{Journals}

Édition électronique

URL : https://journals.openedition.org/mots/609

DOI : $10.4000 /$ mots.609

ISSN : 1960-6001

Éditeur

ENS Éditions

Édition imprimée

Date de publication : 1 juillet 2006

Pagination : $9-22$

ISBN : 2-84788-098-4

ISSN : 0243-6450

Référence électronique

Marcel Burger, Gilles Lugrin, Raphaël Micheli et Stéphanie Pahud, « Marques linguistiques et manipulation. Le cas d'une campagne de l'extrême droite suisse », Mots. Les langages du politique [En ligne], 81 | 2006, mis en ligne le 30 mars 2011, consulté le 22 avril 2022. URL : http:// journals.openedition.org/mots/609; DOI : https://doi.org/10.4000/mots.609 


\section{Marques linguistiques et manipulation. Le cas d'une campagne de l'extrême droite suisse}

Cet article porte sur une annonce ${ }^{1}$ émanant d'un « Comité contre les naturalisations en masse » et parue dans la presse suisse en septembre 2004. L'annonce s'inscrit dans un contexte politique sensible. En effet, le peuple suisse est amené à voter sur des projets de naturalisation facilitée des étrangers de deuxième et troisième générations. Dans leur majorité, médias et politiques ont condamné la dimension manipulatrice de cette annonce, mais sans la (dé)montrer : tout se passe comme si l'accusation de manipulation se suffisait à elle-même et n'avait pas à être appuyée sur des éléments tangibles. II s'agit, dans cet article, d'aller au-delà de tels jugements non étayés qui disqualifient a priori le discours d'extrême droite: le propos de notre analyse est de décrire et d'identifier précisément quelques procédés discursifs de la manipulation. Nous tentons d'éclairer les enjeux communicationnels du texte en nous intéressant à l'articulation de quelques faits iconiques et langagiers significatifs.

Le Comité contre les naturalisations en masse est dirigé par un parlementaire issu de l'Union démocratique du centre (UDC). À l'instar d'autres partis d'extrême droite en Europe, l'UDC suisse promeut un nationalisme exacerbé, notamment manifeste dans ses positions restrictives en matière de politique d'immigration. Si elle se montre résolument hostile aux projets de naturalisation facilitée soumis à la votation populaire, l'UDC nie cependant être à l'origine de la création et du financement du comité. Celui-ci fait publier l'annonce dans l'espace publicitaire des médias de la presse écrite. Comme certains journaux refusent la publication pour des raisons déontologiques, il distribue alors son texte gratuitement dans toutes les boites aux lettres de Suisse romande. Ces évènements déclenchent une polémique qui entraine des prises de position de différents acteurs politiques et d'instances institutionnelles. Le 26 septembre 2004, le peuple rejette les deux projets de naturalisation facilitée, à 56,8\% pour le cas des étrangers de deuxième génération et à 51,6 \% pour le cas des étrangers de troisième génération.

\section{L'annonce est reproduite à la fin du présent article (voir infra).}


Notre analyse s’intéresse à trois dimensions qui déterminent la lecture du texte et, par conséquent, sa portée sur les destinataires. Il est d'abord question des actes de langage que le texte accomplit. Il s'agit ensuite de saisir les principales étapes de la progression argumentative du texte. Nous observons de quelle manière l'argumentation s'emploie, dans un premier temps, à étayer une affirmation de causalité. Nous montrons par quels moyens elle parvient, dans un second temps, à mettre ce raisonnement causal au service d'une conclusion injonctive. Enfin, nous nous intéressons aux diverses représentations du discours d'autrui et à leur dimension persuasive. Il est remarquable que celles-ci ne se limitent pas à un matériau strictement verbal, mais incluent également des chiffres, des pourcentages et des graphiques. Notre hypothèse est que ces représentations du discours d'autrui participent de l'institution d'une scénographie scientifique.

\section{Les actes de langage accomplis par le texte}

Dans la lignée des travaux de John Austin (1970), de John Searle (1972) puis de Daniel Vanderveken (1988), la notion d'acte de langage renvoie à la valeur d'action de l'énonciation telle que le texte en conserve des traces. On admet ainsi que le langage modifie des états du monde en causant l'engagement des sujets de la communication. Cet engagement est déterminé par les modes d'articulation des actes de langage au niveau local et au niveau global (Burger, 2002, p. 80-116 ; Adam, 2005, p. 78-84 et 129-135).

\section{Macro-acte de langage}

Au plan global, on observe des textes où domine une valeur directive, du fait qu'ils incitent les destinataires à accomplir un acte, comme dans le cas d'une pétition politique; des textes où domine une valeur promissive, du fait que l'énonciateur s'engage lui-même à accomplir un acte futur, comme dans le cas d'une reconnaissance de dette ; et d'autres encore où domine une valeur représentative parce qu'ils décrivent le monde sur un mode qui n'est pas forcément neutre, comme un journal télévisé. On parle alors de macro-acte de langage pour renvoyer à la valeur actionnelle dominante d'un texte. Celle-ci est souvent construite en tenant compte de l'horizon d'attentes propre au genre et/ou de l'ancrage référentiel du texte.

À ce titre, on peut faire l'hypothèse que le texte que nous analysons accomplit un macro-acte directif, c'est-à-dire qu'il manifeste une finalité actionnelle consistant à engager les destinataires à effectuer un acte futur. On peut avancer trois raisons à l'appui de cette hypothèse. La première est d'ordre langagier. En effet, le détail linguistique montre que le dernier énoncé du texte, bien mis en évidence au plan typographique, explicite une valeur directive : 
(1) Il faut dire $2 \times$ non aux projets de naturalisation le 26 septembre prochain.

Si la position de cet énoncé dans l'espace rédactionnel est importante, le choix des mots ne l'est pas moins. D'une part, cet énoncé est le seul à mettre en scène les destinataires-citoyens dans un rôle d'agent. D'autre part, on observe que ceux-ci ne sont pas pris à partie sur un mode direct et individualisé (comme cela serait le cas avec «vous direz» ou "vous devez dire »). Au contraire, ils sont intégrés, par la tournure impersonnelle «il faut dire », à un collectif non spécifié. Quant à l'engagement proprement dit, la tournure impersonnelle et le verbe falloir non seulement lui confèrent un caractère de nécessité, mais semblent aussi l'inscrire dans un ordre de détermination quasi naturel. En d'autres termes, les destinataires-citoyens ne sauraient raisonnablement voter autrement qu'il leur est recommandé. Ainsi, la matérialité langagière contribue pour une part à une interprétation globalement directive du texte.

Cependant, cette valeur actionnelle globale est aussi accréditée par la dimension générique du texte. En effet, l'annonce adressée aux citoyens par un organisme politique dans un contexte électoral suppose une finalité incitative. Autrement dit, on s'attend à lire l'exposé d'une opinion à laquelle il est demandé de souscrire ponctuellement. À ce titre, la prise en compte de l'ancrage référentiel du texte constitue la troisième raison à l'appui de l'hypothèse d'un macro-acte directif. Une votation appelle de la part de chaque citoyen un acte consistant à accepter, à refuser ou à s'abstenir de décider d'une mesure modifiant l'organisation de l'espace public. Dans ce contexte référentiel particulier, la mention « 2 x non » aurait même suffi à communiquer une valeur directive. Dans le même ordre d'idées, l'ancrage temporel précis - « le 26 septembre prochain »-achève de spécifier l'action visée.

\section{Micro-actes de langage}

Cela étant dit, la valeur actionnelle globale d'un texte découle plus fondamentalement de l'articulation des actes au plan local. On parle à ce propos de microactes de langage. Dans notre texte, on observe une alternance réglée d'actes directifs et d'actes représentatifs que, faute de place, nous ne pouvons pas analyser dans le détail. Nous centrons l'attention sur deux moments clés de toute stratégie discursive : le début et la fin du texte. Le texte débute par un acte directif de question ${ }^{2}$ :

(2) Grâce aux naturalisations automatiques les musulmans bientôt en majorité ?

suivi de trois énoncés représentatifs qui acquièrent dès lors une valeur de réponse :

2. Si l'on excepte le signe ponctuant, l'énoncé ne comporte pas de propriétés morpho-syntaxiques typiques de l'interrogation. Cela facilite son glissement potentiel vers l'assertion. On examinera plus loin le caractère finalement peu interrogatif de cet énoncé. 
(3) Le nombre de naturalisations progresse d'année en année dans notre pays.

(4) D’ailleurs, aucun pays ne naturalise autant d'étrangers que la Suisse.

(5) Et d'année en année, la population musulmane vivant dans notre pays augmente.

Ces trois assertions ne se situent pas au même niveau. Les énoncés (3) et (5) semblent amorcer une réponse à la question posée dans le titre. Pour ce qui est de l'énoncé (4) introduit par d'ailleurs, il ne semble à première vue constituer qu'une sorte d'appendice à l'énoncé qui précède, se contentant de rappeler un état de choses avéré que personne n'aurait l'idée de venir mettre en doute. En réalité, comme le souligne Oswald Ducrot, « l'élément sur lequel porte d'ailleurs a toujours une valeur argumentative. C'est ce qui fait que d'ailleurs est impossible dans un contexte non argumentatif, lorsqu'on se contente d'inventorier un certain nombre de faits» (1980, p. 197). L'énoncé introduit par d'ailleurs constitue ainsi un argument décisif à l'appui d'une conclusion. Ici, l'énoncé (4) oriente en effet vers deux conclusions (6) et (7) inférées de (3). Celles-ci restent implicites, mais peuvent être rétablies sous la forme d'appréciations de la politique suisse en matière de naturalisations :

(6) [La politique de naturalisation de la Suisse est généreuse.]

(7) [La politique de naturalisation de la Suisse est très (trop) généreuse.]

L'usage de d'ailleurs a ceci d'avantageux qu'il permet à l'énonciateur d'atténuer le degré de sa prise en charge de l'énoncé et, par là même, d'échapper à l'obligation de se justifier. Lorsqu'il utilise d'ailleurs, l'énonciateur «prétend [...] ne pas utiliser [l'énoncé introduit], mais seulement l'évoquer. [...] Il présente le destinataire comme déjà d'accord (il fait comme s'il prêchait à un converti) » (Ducrot, 1980, p.195 et 222). Ici, l'énonciateur fait comme s'il apportait une information additionnelle qui ferait l'objet d'un consensus, alors qu'il oriente argumentativement vers un jugement de valeur implicite. Dans le même ordre d'idées, le lien opéré entre (4) et (5) par la conjonction et facilite l'inférence d'un contenu (8) :

(8) [La politique de naturalisation de la Suisse est (trop) généreuse envers les musulmans.]

En d'autres termes, c'est bien de l'articulation des trois micro-actes d'assertion qu'émerge une réponse implicite (9), et lourde de connotations négatives, à la question initiale :

(9) [Oui, les musulmans seront bientôt en majorité en Suisse.] 
Cette réponse implicite ${ }^{3}$ constitue le principal motif de l'acte directif d'injonction qui clôt le texte :

(1') Pour empêcher ce bradage du droit de cité suisse, il faut dire $2 \mathrm{x}$ non aux projets de naturalisation le 26 septembre prochain.

Un lien clair apparait ainsi entre le groupement d'actes initiaux et la fin du texte. À ce titre, la clause «pour empêcher ce bradage du droit de cité suisse » exerce une fonction à la fois projective et rétroactive. Elle fournit, d'une part, une raison à l'appui de l'acte directif qui la suit immédiatement et qu'elle légitime. D'autre part, l'usage du démonstratif ce et du terme péjoratif bradage tend à imposer au destinataire une (ré)interprétation de ce qui précède dans le texte. Il est remarquable que le jugement négatif ne fasse pas l'objet d'une prédication, mais soit présupposé : le texte fait habilement l'économie de l'assertion «C'est un bradage du droit de cité suisse », ce qui a pour effet d'en présenter le contenu comme déjà accepté par le destinataire. Il y a là un court-circuit qui vise la création d'un effet d'évidence : il va de soi que la politique de naturalisation soumise au vote est un «bradage du droit de cité suisse ».

\section{La dynamique argumentative du texte}

Après cette brève étude des actes de langage qui structurent le texte au niveau global et au niveau local, on peut s'intéresser plus en détail aux principales étapes de la progression argumentative. On va voir que l'argumentation développe un raisonnement causal projectif qui relie une cause et un effet futurs. Elle cherche ensuite à étayer l'affirmation de causalité par une série d'énoncés apparemment constatifs portant sur l'état actuel du monde. Il s'agit enfin de comprendre comment un tel raisonnement causal peut déboucher sur une conclusion directive.

\section{Un raisonnement causal projectif}

Le titre de l'annonce est un énoncé prédictif, c'est-à-dire une assertion portant sur un état de choses futur. La typographie interrogative ne doit pas être prise pour argent comptant: l'énoncé ne constitue pas une simple demande d'information dont la réponse serait à la charge de l'allocutaire. Bien loin d'être une question dont le locuteur ne préjugerait en rien de la réponse, cet énoncé est unilatéralement orienté vers une réponse positive, d'ailleurs fournie dans

3. La problématique centrale de savoir «qui» donne la réponse, ainsi que le tour de force qui consiste à poser comme équivalentes la population musulmane et la population étrangère demandant la naturalisation, sont abordés plus loin. 
le corps du texte - d'abord sous la forme d'une assertion conditionnelle (" $\mathrm{Si}$ cette évolution se poursuit, les musulmans seront bientôt la majorité dans ce pays »), puis sous celle d'une assertion dont la prise en charge se voit déléguée à Sami Aldeeb, énonciateur présenté comme un expert («Dans vingt ans, ils [les musulmans] seront la majorité »). Par son caractère indirectement assertif, l'énoncé inaugural se rapproche d'une question rhétorique. Celle-ci tend, comme l'a montré Charles Bally, à un renforcement de l'assertion et va même jusqu'à l'investir de valeurs affectives proches de l'exclamation (1951, p. 269).

L'énoncé-titre avance donc une prédiction, mais procède, dans le même temps, à une affirmation de causalité. Il ne se contente pas de prédire la réalisation d'un état futur: il présente celle-ci comme l'effet résultant d'une cause identifiable. On s'arrêtera un instant sur la locution prépositionnelle grâce à qui marque la relation causale. Cette locution a pour particularité d'introduire la cause d'un évènement que l'énonciateur présente comme désirable ou heureux. On peut penser qu'il s'agit d'un usage ironique. Le locuteur feint de donner la cause d'un évènement heureux et, par là même, de porter un jugement positif sur ladite cause.

L'énoncé-titre se rapproche de l'une des formes du raisonnement causal (causal reasoning) décrites par Douglas Walton (1995, p. 140) : "The argument from cause to effect concludes that a particular event or state of affairs will or might occur on the grounds that another event will or might occur that will or might cause it. » Le propos de Walton met en évidence le caractère projectif et, par là, fort incertain d'un tel raisonnement. On notera que la cause (E1) relève, tout comme son effet supposé (E2), d'une projection dans le futur : les « naturalisations automatiques » sont celles qui seraient pratiquées au cas où le peuple donnerait son accord aux deux projets de loi. L'énoncé-titre fonde ainsi l'hypothèse de la réalisation probable d'un état de choses E2 (" les musulmans bientôt en majorité ? ») sur une autre hypothèse - celle de la réalisation probable d'un état de choses E1, censé causer E2. En d'autres termes, il conclut d'une cause à un effet, alors que ni l'une ni l'autre ne sont avérées au moment de l'énonciation.

\section{Un étayage de l'affirmation de causalité?}

Examinons à présent le rôle du chapeau dans la dynamique argumentative :

(3) Le nombre de naturalisations progresse d'année en année dans notre pays.

(4) D'ailleurs, aucun autre pays ne naturalise autant d'étrangers que la Suisse.

(5) Et d'année en année, la population musulmane vivant dans notre pays augmente.

Ce qui est en jeu ici, c'est de montrer que l'affirmation causale du titre, loin de ne constituer qu'une projection dans l'avenir, est déjà en partie valable dans 
l'état actuel du monde. On quitte le raisonnement causal projectif, dont la cause comme l'effet relèvent de l'hypothèse, pour passer à une série de constats qui portent sur un état de choses donné comme effectif et empiriquement accessible. Alors que le titre évoquait deux états possibles et suggérait que l'un causerait presque immanquablement l'autre, le chapeau se focalise sur des processus apparemment en cours au moment de l'énonciation. Il semble avoir pour rôle d'étayer la prédiction du titre par une observation de la réalité et des relations causales que l'on peut y déceler. On remarque cependant que la relation causale entre, d'une part, la «progress[ion]» du «nombre de naturalisations » et, d'autre part, l'« augment[ation] » de la " population musulmane » en Suisse n'est pas directement affirmée. On ne trouve pas de connecteur ou d'expression marquant la causalité de façon explicite: tout au plus observet-on, en tête du troisième énoncé, un et. Cette conjonction permet certes, lorsqu'elle coordonne des propositions, d'exprimer des relations variables, parmi lesquelles la conséquence. Néanmoins, la relation de causalité n'est ici pas tant affirmée qu'elle n'est impliquée par la symétrie entre les énoncés (3) et (5). La perception d'un lien causal repose certainement sur l'effet de ressemblance induit par le jeu des parallélismes. Les deux verbes progresser et augmenter peuvent être indexés à une même isotopie de la croissance. On notera également la récurrence des compléments circonstanciels de temps ("d'année en année ») et de lieu ("dans notre pays»). La symétrie des deux énoncés et le jeu des parallélismes qui s'y déploie rendent ainsi très difficile une lecture qui envisagerait les phénomènes décrits indépendamment l'un de l'autre.

Il importe de relever que le discours prend une allure constative, comme s'il n'argumentait pas et se contentait d'enregistrer des phénomènes en cours. On peut emprunter une formule dont Christian Plantin fait usage dans une réflexion sur l'argumentation dite "par la cause »: «La formulation de l'argument n'évoque explicitement qu'une série de coïncidences, mais il ne fait pas de doute qu'elle repose sur une affirmation de causalité. » (1990, p. 220)

\section{De l'affirmation de causalité à l'injonction}

Le titre et le chapeau procèdent, de façon explicite ou implicite, à une affirmation de causalité. Comment, dès lors, passer d'un raisonnement de type causal à un acte directif qui inciterait le destinataire à adopter un certain comportement? L'établissement d'une relation causale ne livre en lui-même aucune conclusion sur le plan pratique : prétendre que telle cause engendrera tel effet ne suffit pas à indiquer de façon univoque ce qu'il convient de faire. Il s'agit à présent de comprendre comment l'argumentation de l'annonce met l'affirmation causale au service d'une conclusion pratique. Le passage à une telle conclusion exige qu'un jugement de valeur soit préalablement porté sur l'effet. Or, si l'on considère l'annonce dans son ensemble, on peine à trouver un jugement 
de valeur négatif qui serait explicitement posé et directement pris en charge par l'énonciateur. Intéressons-nous plus précisément à la deuxième section de l'annonce ("La majorité dans 20 ans? ?). L'énonciateur cite d'abord un expert, qui valide l'énoncé prédictif du titre: "Dans vingt ans, ils [les musulmans] seront la majorité. Il y aura bientôt plus de musulmans que de chrétiens en Suisse ». L'énonciateur enchaine ensuite : «C'est bien là que réside le problème, car “les Musulmans placent leur religion au-dessus de nos lois". „ L'appréciation négative du contenu de la prédiction est à la charge de l'énonciateur. En revanche, et c'est essentiel, la justification de cette appréciation négative ne l'est pas. Par l'usage du discours rapporté direct et des guillemets, l'énonciateur se décharge de la proposition justificative introduite par car : celle-ci relève de la responsabilité de l'autorité invoquée.

Même peu assumé, ce jugement de valeur sur l'effet permet d'embrayer sur une conclusion pratique. Pour bien comprendre l'enchainement, revenons très brièvement sur les principales étapes de l'argumentation que nous avons pu identifier. L'argumentation amorce, dans un premier temps, un raisonnement causal projectif: elle affirme la réalisation probable d'un état de choses ("Les musulmans bientôt en majorité?») et présente celui-ci comme l'effet d'une cause encore non avérée (l'acceptation des projets de loi sur la naturalisation facilitée). L'argumentation revient, dans un second temps, à la situation présente, probablement dans le but d'étayer la relation de causalité. En fait d'étayage, c'est plutôt à la recherche d'un effet d'évidence que l'on assiste, notamment par le jeu des parallélismes entre les énoncés. Une fois la relation de causalité postulée, il reste, on vient de le voir, à porter un jugement de valeur sur l'effet. L'énonciateur le fait, mais du bout des lèvres, en déléguant la majeure partie de la responsabilité énonciative à un autre énonciateur présenté comme un expert. L'établissement supposé d'une relation causale et le jugement de valeur négatif porté sur l'effet permettent, en dernier lieu, de formuler une incitation à agir. C'est l'objet du dernier énoncé du texte, que nous avons préalablement décrit comme un acte directif (voir supra):

(1) Pour empêcher ce bradage du droit de cité suisse, il faut dire 2 x non aux projets de naturalisation le 26 septembre prochain.

Cet énoncé encapsule à la fois le jugement de valeur sur l'effet (par le biais du terme péjoratif bradage) et l'incitation à agir. Le principe est que l'action sur une cause se répercute sur son effet : on vise, dans un tel esprit, à agir sur l'effet en agissant sur sa cause. Ceci présuppose la croyance en une «proportionnalité de la cause à l'effet: selon que nous renforcerons/atténuerons la cause, l'effet se trouvera renforcé/atténué » (Plantin, 1990, p. 224). Ici, l'énonciateur incite l'allocutaire à atténuer l'effet jugé indésirable en s'attaquant à sa cause. 


\section{Les représentations du discours d’autrui}

Comme on l'a déjà entrevu, l'énonciation de l'annonce est loin d'être homogène. Plusieurs sources énonciatives externes sont convoquées et mises en scène. Sont cités, d'une part, les propos d'un expert en matière de droit musulman, Sami Aldeeb. Sont donnés en référence, d'autre part, des chiffres, tirés pour les uns de l'Office de la statistique du canton de Zurich ainsi que de l'Office fédéral de la statistique, et, pour les autres, d'un site internet (www.pikom.ch). Fonder une argumentation sur des paroles autres permet à l'énonciateur de lui conférer plus de poids, mais aussi de ne pas avoir à assumer seul la prise en charge de ses aspects les plus contestables, voire les plus mensongers.

\section{Recours à la citation d'autorité}

Sami Aldeeb, responsable du droit arabe et musulman à l'Institut suisse de droit comparé de Lausanne, est la principale source externe à laquelle l'annonce fait appel. Les fragments de discours qui sont prêtés à l'expert sont repris littéralement d'une interview parue quelques mois plus tôt dans un quotidien suisse alémanique, le Blick. Intitulé «Muslime stellen ihre Religion über unser Gesetz» ("Les musulmans placent leur religion au-dessus de nos lois»), l'article en question avait créé la polémique, au détriment du principal intéressé qui était d'ailleurs opposé à sa publication.

Décontextualiser puis recontextualiser un énoncé revient immanquablement à en modifier le sens: tout déplacement produit une valeur neuve et entraine une transformation qui affecte tout à la fois le signifié du texte cité et celui du texte d'accueil. La citation des propos de Sami Aldeeb ne fait pas exception à cette règle : décontextualisées, ses paroles peuvent prendre une connotation islamophobe, alors qu'elles sont, à en croire le principal intéressé4 ${ }^{4}$, le fruit d'une pensée prônant l'intégration. La stratégie mise en œuvre par le texte est toutefois plus complexe. Elle consiste à placer la critique des musulmans dans la bouche d'un énonciateur dont le statut professionnel comme le nom tendent précisément à désamorcer toute présomption d'islamophobie. En jouant sur les inférences que le lecteur est susceptible de tirer de son patronyme $e^{5}$, le texte présente Sami Aldeeb comme un énonciateur libre de tout préjugé négatif envers les musulmans. L'énoncé "Les Musulmans placent leur religion audessus de nos lois » n'en acquiert dès lors que plus de poids : il reçoit en effet la caution d'un énonciateur dont le point de vue ne parait souffrir d'aucun biais.

4. Entretien avec les auteurs (université de Lausanne, aout 2005).

5. Inférences trompeuses, puisque l'universitaire est en l'occurrence un chrétien d'origine palestinienne. 
La critique des musulmans ne semble ici pas procéder d'une hostilité viscérale de l'énonciateur à leur égard, ce qui en affaiblirait la portée: en insistant sur le domaine de spécialisation de l'énonciateur («droit arabe et musulman»), le texte pare en effet cette critique des atours de l'objectivité et de la modération que l'on attend d'une analyse académique. L'habileté du texte réside dans sa capacité à déléguer la prise en charge de la dévalorisation des musulmans et de leur modus vivendi à un énonciateur que le lecteur aurait justement tendance à placer au-dessus de tout soupçon en matière d'islamophobie.

\section{L'infographie et l'institution d'une scénographie scientifique}

L'infographie fait, elle aussi, appel à des références d'autorité. Elle est constituée de trois courbes, légèrement superposées de manière à induire un effet de profondeur. La courbe centrale porte sur l'évolution du nombre de naturalisations entre 1990 et 2002. Elle est entourée de deux courbes portant l'une sur la «Part des musulmans à la population du canton de Zurich » et l'autre sur la «Part en \% des musulmans à la population totale de la Suisse ». La superposition des trois courbes n'est pas le fruit du hasard: elle traduit visuellement l'amalgame qui, dans la partie rédactionnelle de l'annonce, fait de l'augmentation des naturalisations une cause ayant pour effet l'augmentation de la population musulmane en Suisse (comme analysé précédemment).

Cet agencement topographique s'accompagne d'une manipulation des chiffres représentés par les courbes. En effet, la courbe médiane de l'infographie, dont les données sont tirées du site Pikom, est la seule en rapport avec le sujet de la votation et, surtout, la seule à ne pas présenter de chiffres discutables, voire erronés. Il est cependant intéressant de noter que, contrairement à ce que suggère son nom, «Parteiunabhängiges Informationskomitee» ("Comité d'information indépendant de tout parti »), Pikom abrite des représentants de I'UDC, qui font par ce biais leur autopromotion.

Les données de la courbe placée en arrière-plan sont attribuées à l'Office de la statistique du canton de Zurich. On remarque que cette courbe présente des valeurs absolues. Or, des pourcentages permettraient de pondérer le nombre de musulmans présents dans le canton de Zurich en fonction de la population totale et constitueraient de fait des données plus représentatives. Le choix de Zurich n'est à cet égard pas anodin, puisqu'il s'agit du canton le plus peuplé de Suisse (1 200000 habitants en 2000). Le chiffre de 67000 musulmans dans ce canton représente en réalité $5,5 \%$ de la population du canton. Ajoutons enfin que sur les 67000 musulmans habitant dans le canton de Zurich, 9519 sont Suisses ${ }^{6}$. Ce canton est l'un de ceux qui connaissent par ailleurs, après ceux de Genève et

6. Voir le site de l'Office fédéral de la statistique : http://www.bfs.admin.ch/bfs/portal/fr/index/ themen/bevoelkerung/uebersicht/blank/medienmitteilungen.Document.49121.html.

18 - Suisse, laboratoire politique européen? 
de Bâle, l'augmentation la plus massive d'étrangers, toutes confessions confondues $^{7}$. Le canton d'Uri aurait par exemple présenté des chiffres tout autres. Le choix de se centrer sur le canton de Zurich et de présenter des valeurs absolues (et non des pourcentages) permet de présenter une courbe alarmiste ${ }^{8}$.

La courbe placée au premier plan de l'annonce est de loin la plus intéressante, par sa position, par les chiffres alarmistes qu'elle présente ainsi que par sa dimension manipulatrice. Cette courbe est, avec le titre et la consigne de vote qu'elle permet précisément de relier, l'une des trois zones mises en évidence par l'agencement de l'annonce. La linéarité de la courbe masque d'autre part son caractère composite. La coprésence sur la courbe d'astérisques simples et doubles, peu repérables à moins d'une lecture particulièrement attentive, sème la confusion. Non seulement il n'est pas évident de repérer que certains chiffres sont des extrapolations, mais encore il n'est pas dit si ces extrapolations sont du ressort de l'Office fédéral de la statistique ou de celui du Comité contre les naturalisations en masse. Le coup de force de l'annonce tient justement à cette confusion. En effet, l'Office est cité comme source et l'on tend à lui attribuer l'ensemble des chiffres du graphique, y compris les extrapolations. Cependant, comme ce dernier l'a expliqué dans une lettre officielle9, seuls les deux premiers chiffres $(1990,2000)$ proviennent des recensements fédéraux de la population. Les autres chiffres, «cité[s] d'une manière qui prête à confusion $»^{10}$, sont des extrapolations dénuées de fondement scientifique, puisque si l'on suit cette logique, il y aurait $144 \%$ de musulmans en 2050 . Cette progression exponentielle de la part de musulmans par rapport à la population totale de la Suisse n'est d'ailleurs pas fortuite dans un contexte où l'extrémisme islamiste est régulièrement mis sur le devant de la scène médiatique. L'infographie amalgame ainsi des chiffres alarmistes sur la progression du nombre de musulmans et la question des naturalisations, passant sous silence le fait que les musulmans de Suisse ne sont pas tous étrangers et que tous les étrangers ne sont pas musulmans.

\section{Trois formes de manipulation}

En fin de compte, l'annonce semble recourir aux trois formes de manipulation identifiées par Philippe Breton (1997). Manipulation des affects, d'abord:

7. Voir les chiffres de l'Observatoire suisse de la santé («Part de la population étrangère et de la population suisse dans la population résidante permanente », source : http://www.obsan.ch/ monitoring/statistiken/1_1_2/2001/f/).

8. Les chiffres de l'Observatoire suisse de la santé présentent par contraste une analyse différente de la proportion de la population étrangère et suisse au sein de la population résidante permanente entre 1970 et 2001 . On constate qu'on est loin de la croissance exponentielle dont l'annonce fait état lorsqu'elle évoque les musulmans.

9. Se référer, ici encore, au site de l’Office fédéral de la statistique (voir supra, note 6).

10. Ibid. 
l'annonce exploite chez les citoyens la peur du terrorisme islamiste et plus généralement la confusion entre islam, islamisme et terrorisme, que les médias suisses entretiennent depuis les attentats du 11 septembre et la guerre en Irak (voir Burger, à paraitre). À ce titre, l'UDC surfe sur une vague d'islamo-méfiance qui touche la Suisse et plus globalement l'Europe (voir Schneuwly et Lathion, 2003 ; Rebetez et Lorenzi, 2003). Il s'agit bien là d'un amalgame affectif, lequel " consiste à rendre acceptable une opinion en construisant un message qui est un mélange de cette opinion, sans discussion de son contenu, avec un élément extérieur, de l'ordre de l'affect, sans rapport immédiat avec cette opinion, mais considéré, lui, comme susceptible de sensibiliser l'auditoire dans un sens favorable» (Breton, 1997, p. 91). L'annonce vise en priorité un engagement motivé par des réactions émotionnelles des citoyens.

Manipulation des faits, ensuite : la désinformation a souvent pour principe de mélanger subtilement de vraies et de fausses informations. Or, « toute l'habileté technique de la désinformation tient justement dans le mécanisme qui permet de travestir une information fausse en une information "vraie" qui soit parfaitement crédible et qui oriente l'action de celui qui la reçoit dans un sens qui lui est défavorable »(Breton, 1997, p. 66). Le recours à la scénographie scientifique, doublé de la combinaison de chiffres attestés et d'extrapolations extravagantes - mais présentées comme émanant d'une autorité incontestable et légitime -, est symptomatique d'une volonté manifeste de désinformer pour mieux manipuler. L'énonciateur cherche à accroitre sa crédibilité par quelques chiffres qui ne prêtent pas à discussion, puisqu'ils sont placés sous l'égide d'une source officielle.

Manipulation cognitive, enfin : « La différence entre l'amalgame affectif, que nous avons déjà évoqué, et l'amalgame cognitif est que, dans un cas, il y a une association non fondée avec un élément affectif, un sentiment, une image, alors que, dans l'autre, on suggère un lien de causalité, qui n'est pas, lui non plus, fondé. » (Breton, 1997, p. 120-121) L'amalgame est ici exemplaire dans le sens où il est à la fois affectif, parce que sensible, et cognitif : tant les énoncés du chapeau (voir supra) que la superposition des trois courbes (idem) suggèrent avec insistance, mais sans jamais le démontrer, un lien causal entre le nombre de naturalisations et le nombre de musulmans vivant en Suisse. C'est, à notre sens, ce lien causal infondé que tente d'imposer avec force l'ensemble du texte. 


\section{Grâce aux naturalisations automatiques Les Musulmans bientôt en majorité?}

Le nombre de naturalisations progresse d'année en année dans notre pays. D'ailleurs, aucun autre pays ne naturalise autant d'étrangers que la Suisse.

Et d'année en année, la population musulmane vivant dans notre pays augmente.

\section{La proportion de Musulmans double tous les dix ans en Suisse}

Aucune communauté religieuse n'augmente aussi rapidement que calle des Musulmans. En 1970, 4000 habitants du canton de Zurich s'étaient déclarés comme appartenant à I'islam. II s'agissait donc d'un habitant sur 280. En 1990, les Musulmans étaient dejaja 30700 dans le canton de Zurich. Ce chiffre a doublé juscu'en 2000 oủ l'on comptait quolque 67000 Musulmans dans ce canton. Ainsi, un habitant sur 19 est aujourd'hui Musulman.

La situation est la mème au niveau national. L'orfice fédéral de la statistique relève d'ailleurs aussi la croissance particulierement forte de la communauté isłamioue. Alors que 152'200 Musulmans viraient en Sulsse en 1990, ils étaient plus de 310000 en l'an 2000 Donc au niveau suisse aussi, on reletve un doublement en l'espace de dix ans. Si cette évolution se poursuit, les Musulmans seront bientót la majorité dans ce pays. Le taux des naissances est en effet sensiblement plus élevé dans les familles musulmanes que dans les familles chrétiennes.

\section{La majorité dans $\mathbf{2 0}$ ans?}

Sami Addeeb, responsable du droit arabe et musulman à I'Institu: sulsse de droit comparé à Lausanne, a une vision encore plus drastique de la situation s La proportion de Musulmans en Suisse triple tous les dix ans. Aujourd'hul, $310^{\circ} 000$ Musulmans vivent legalement

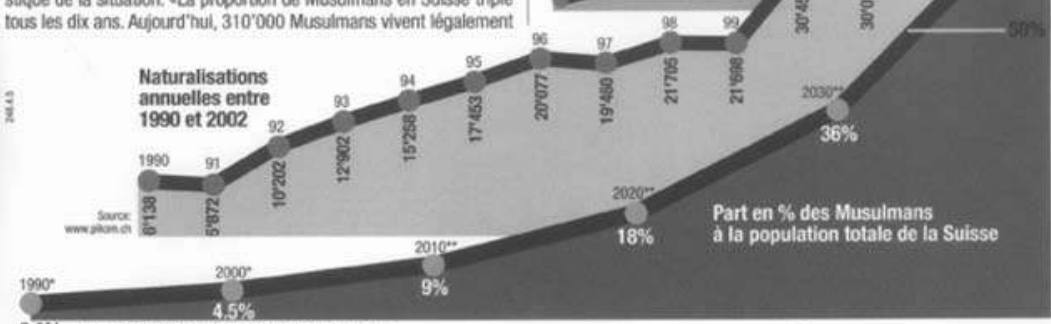

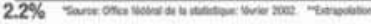

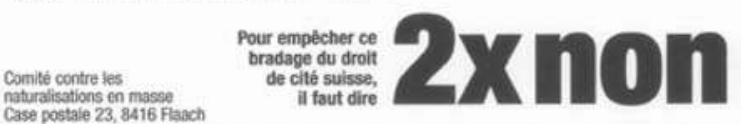

aux projets de naturalisation le 26 septembre prochain. 


\section{Références}

ADAM J.-M., 2005, La linguistique textuelle. Introduction à l'analyse textuelle des discours, Paris, Armand Colin, $234 \mathrm{p}$.

AUSTIN J.-L., 1970, Quand dire, c'est faire, Paris, Le Seuil, 203 p.

BALLY C., 1951, Traité de stylistique française, Paris, Klincksieck, $331 \mathrm{p}$.

BRETON P., 1997, La Parole manipulée, Paris, La Découverte, 221 p.

BURGER M., 2002, Les manifestes : paroles de combat. De Marxà Breton, Paris, Delachaux et Niestlé, 352 p. (Un compte rendu de cet ouvrage est paru dans Mots. Les langages du politique, $\mathrm{n}^{\circ} 80$, mars 2006, p. 123.)

_ à paraitre, "The media and the war : the role of titles and subtitles in the construction of other negative identities», A. Hodges et C. Nillep éd., Discourse, War and Terrorism, Amsterdam, Philadelphia, John Benjamins.

DUCROT O., 1980, Les mots du discours, Paris, Minuit, $241 \mathrm{p}$.

PLANTIN C., 1990, Essais sur l'argumentation, Paris, Kimé, 351 p.

REBETEZ A. et LORENZI M. (Entretien avec-), 2003, « Peut-on parler d'islamophobie dans les médias suisses romands ? ", Boèce, $\mathrm{n}^{\circ}$ 6, p. 21-38.

SEARLE J., 1972, Les actes de langage. Essai de philosophie du langage, Paris, Hermann, $260 \mathrm{p}$.

SCHNEUWLY M. et LATHION S., 2003, "Panorama de l'islam en Suisse », Boèce, $\mathrm{n}^{\circ} 6$, p. 7-20.

VANDERVEKEN D., 1988, Les actes de discours, Bruxelles, Mardaga, $226 \mathrm{p}$.

WALTON D., 1995, A Pragmatic Theory of Fallacy, Tuscaloosa, Alabama University Press, $302 \mathrm{p}$. 